\title{
Evolutionary Undersampling for Extremely Imbalanced Big Data Classification under Apache Spark
}

\author{
I. Triguero, M. Galar, D. Merino, J. Maillo, H. Bustince, F. Herrera
}

\begin{abstract}
The classification of datasets with a skewed class distribution is an important problem in data mining. Evolutionary undersampling of the majority class has proved to be a successful approach to tackle this issue. Such a challenging task may become even more difficult when the number of the majority class examples is very big. In this scenario, the use of the evolutionary model becomes unpractical due to the memory and time constrictions. The divide-and-conquer approaches based on MapReduce paradigm have already been proposed to handle these types of problems by dividing data into multiple subsets. However, in extremely imbalanced cases, these models may suffer from a lack of density from the minority class in the subsets considered. Aiming at addressing this problem, in this contribution we provide a new big data scheme based on the new emerging technology Apache Spark to tackle highly imbalanced datasets. We take advantage of its inmemory operations to diminish the effect of the small sample size. The key point of this proposal lies on the independent management of majority and minority class examples, allowing us to keep a higher number of minority class examples in each subset. In our experiments we analyze the proposed model with several data sets with up to 17 million instances. The results show the goodness of this evolutionary undersampling model for extremely imbalanced big data classification.
\end{abstract}

\section{INTRODUCTION}

In the recent years, the amount of information that can be automatically gathered is inexorably growing in multiple fields such as bioinformatics, social media or physics. Thus, new class of data mining techniques that can take advantage of this voluminous data to extract valuable knowledge are required. This research topic is referred to under the term: big data [1]. Big data learning poses a significant challenge to the research community because standard data mining models cannot deal with the volume, diversity and complexity that these data bring up [2]. However, the newly arisen cloud platforms and parallelization technologies provide one with a perfect environment to tackle this issue.

The MapReduce framework [3], and its open-source implementation in Hadoop [4], were the first alternatives to

This work was supported by the Research Projects TIN2011-28488, TIN2013-40765-P, P10-TIC-6858 and P11-TIC-7765. I. Triguero holds a BOF postdoctoral fellowship from the Ghent University.

I. Triguero is with the Department of Internal Medicine of the Ghent University, 9052 Zwijnaarde, Belgium. E-mails: \{isaac.triguero@irc.vibugent.be

D. Merino, J. Maillo and F. Herrera are with the Department of Computer Science and Artificial Intelligence of the University of Granada, CITICUGR, Granada, Spain, 18071. E-mails: dmerino76@gmail.com, \{jesusmh, herrera\}@decsai.ugr.es

M. Galar and H. Bustince are with the Department of Automatics and Computation, Universidad Pública de Navarra, Campus Arrosadía s/n, 31006 Pamplona, Spain. E-mails: \{mikel.galar, bustince\}@unavarra.es handle data-intensive applications, which rely on a distributed file system. The development of Hadoop-based data mining techniques has been widely spread [5], [6], because of its fault-tolerant mechanism (recommendable for timeconsuming tasks) and its ease of use [7]. Despite its popularity, researchers have encountered multiple limitations in Hadoop MapReduce to develop scalable machine learning tools [8]. Hadoop MapReduce is inefficient for applications that share data across multiple phases of the algorithms behind them, including iterative algorithms or interactive queries. Several platforms have recently emerged to overcome the issues presented by Hadoop MapReduce [9], [10]. Apache Spark [11] highlights as one of the most flexible and powerful engines to perform faster distributed computing in big data by using in-memory primitives. This platform allows us to load data into memory and query it repeatedly, making it very suitable for algorithms that use data iteratively.

The class imbalance problem is challenging when it appears in data mining tasks such as classification [12]. Focusing on two-class problems, the issue is that the positive instances are usually outnumbered by the negative ones, even though the positive one is usually the class of interest [13]. This problem is presented in a large number of real-world problems [12]. Furthermore, it comes along with a series of difficulties such as small sample size, overlapping or small disjuncts [14]. In this scenario, one focuses on correctly identifying the positive examples, but affecting the least to the negative class identification. Various solutions have been developed to address this problem, which can be divided into three groups: data sampling, algorithmic modifications and cost-sensitive solutions. These approaches have been successfully combined with ensemble learning algorithms [15].

Evolutionary undersampling (EUS) [16] falls in the category of data sampling strategies, where the aim is to balance the original dataset. In this case, the balancing is done by undersampling, that is, reducing the number of negative class examples. Differently from random undersampling where the focus is put on balancing the dataset, EUS has a two-fold objective. 1) To create the balanced dataset; 2) To increase the overall performance over both classes of the problem. In order to do so, a supervised balancing procedure is carried out using a genetic algorithm. Once the dataset is balanced, any standard classifier can be used to build a model that should be able to equally distinguish both classes of the problem. This technique is very powerful when dealing with standard imbalanced problems, however, when shifting to a large-scale context it becomes unfeasible since the search space increases exponentially with the number of instances 
of the problem.

In [17], we proposed a MapReduce-based EUS scheme to tackle imbalance big data problems. This model splits the dataset into multiple chunks that are processed in different nodes (mappers) in such a way that EUS can be applied concurrently. Even though this model can scale to very large datasets, it may suffer from the small sample size problem. Within this divide-and-conquer procedure, a high number of maps implies having a considerably smaller amount of minority class examples in each one, what amplifies the lack of density problem.

In this work, we propose a big data scheme for extremely imbalance problems implemented under Apache Spark, which aims at solving the lack of density problem in our previous model. We aim to exploit the flexibility provided by Spark, using other in-memory operations that alleviate the consumption costs of existing MapReduce alternatives. Multiple parallel operations compose the proposed framework. First, the whole training dataset is split into chunks, and the positive examples are extracted from it. Then, we broadcast the positive set, so that, all the nodes have a single in-memory copy of the positive samples. For each chunk of the negative data, we aim to obtain a balanced subset of data using a sample of the positive set. Later, EUS is applied to reduce the size of both classes and maximize the classification performance, obtaining a reduced set that is used to learn a model. Finally, the different models are combined to predict the classes of the test set. The source code of this model as well as the ones used in the experiments of this work are available at GitHub ${ }^{1}$.

The paper is structured as follows. Section II provides background information about imbalanced classification, EUS and MapReduce. Section III describes the proposal. Section IV analyzes the empirical results. Finally, Section $\mathrm{V}$ summarizes the conclusions.

\section{BACKGROUND}

This section briefly describes the topics used in this paper. First, the MapReduce paradigm and the Spark framework are introduced in Section II-A. Then, the state-of-the-art on imbalanced big data classification is presented (Section II-B), and the EUS algorithm is recalled (Section II-C).

\section{A. MapReduce and Hadoop/Spark Frameworks}

The MapReduce programming paradigm [3] is a scalable data processing tool designed by Google in 2003. It was designed to be part of the most powerful search-engine on the Internet, but it rapidly became one of the most effective techniques for general-purpose data intensive applications.

MapReduce is based on two user-defined operations: Map and Reduce. The Map function reads the raw data as keyvalue pairs <key,value $>$, and transforms them into a set of intermediate <key',value'> pairs. Both key and value types are defined by the user. Then, MapReduce generates multiple lists with all the values with the same key

\footnotetext{
${ }^{1}$ https://github.com/triguero/EUS-BigData
}

$<$ key',list(value') $>$ (shuffle phase). Finally, the Reduce function takes the grouped output from the maps and aggregates it into a smaller set of pairs <key",value" $>$. Figure 1 shows a flowchart of MapReduce.

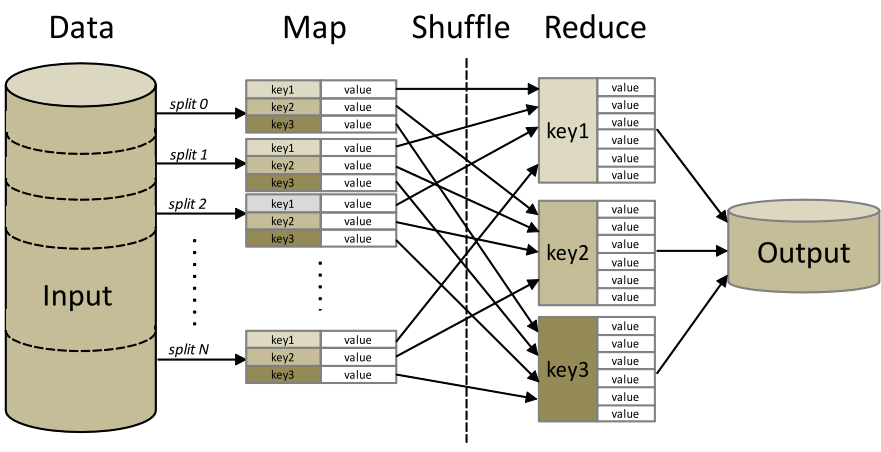

Fig. 1: Data flow overview of MapReduce

Apache Hadoop [18] is the most popular open-source implementation of MapReduce. It is widely used because of its performance, open source nature, installation facilities and its distributed file system (Hadoop Distributed File System, HDFS). Despite its popularity, Hadoop and MapReduce cannot deal with online or iterative computing, producing significant computational costs to reuse the data.

Apache Spark is a novel solution large-scale data processing to solve the drawbacks of Hadoop. Spark is part of the Hadoop Ecosystem and it uses the HDFS. This framework proposes a set of in-memory primitives, beyond the standard MapReduce, aiming at processing data more rapidly on distributed environments. Spark is based on Resilient Distributed Datasets (RDDs), a special type of data structure used to parallelize the computations in a transparent way. These parallel structures let us persist and reuse results efficiently, since they are cached in memory. Moreover, they also let us manage the partitioning to optimize data placement, and manipulate data using transparent primitives.

\section{B. Imbalanced classification in the Big Data context}

A two-class classification dataset is imbalanced when it contains more instances from one class than from the other one. How to measure the performance of classification algorithms is a key issue in this framework, where the accuracy rate (percentage of correctly classified examples) is no longer valid. The most commonly considered alternatives in this scenario are the Area Under the ROC Curve (AUC) and the g-mean. The AUC (Area Under the ROC-Curve) [19] provides a scalar measurement of how well a classifier can trade off its true positive $\left(\mathrm{TP}_{\text {rate }}\right)$ and false positive rates $\left(\mathrm{FP}_{\text {rate }}\right)$. A popular approximation [12] of this measure is given by

$$
A U C=\frac{1+\mathrm{TP}_{\text {rate }}-\mathrm{FP}_{\text {rate }}}{2} .
$$

Otherwise, the g-mean is obtained by computing the geometric mean between the true positive rates and true 
negative rates $\left(\mathrm{TN}_{\text {rate }}\right)$ obtained by the classifier:

$$
\mathrm{g}-\text { mean }=\sqrt{\mathrm{TP}_{\text {rate }} \cdot \mathrm{TN}_{\text {rate }}}
$$

The interest of this measure resides in the fact that equal weights are assigned to the classification accuracy over both classes. Both measures are interchangeably and extensively used in numerous experimental studies with imbalanced datasets [12], [16].

Big data solutions for classification problems can be affected by the presence of class imbalance. They can even worsen the problem if they are not properly designed. For example, an imbalanced dataset distributed across different nodes will maintain the imbalance ratio in each one, but it will have an even lower sample size due to the original division procedure. As a result, data subsets will be more affected by the small sample size problem than the original one, which is known to hinder classifier learning [12]. Therefore, meaningless classifiers can be learned in each node if they are treated independently without taking this issue into account first.

In [20], a set of data level algorithms to address imbalanced big data classification where tested (random under/oversampling and SMOTE). After applying these preprocessing mechanisms the Random Forest classifier [23] was applied. In other respects, the authors of [24] developed a fuzzy rule based classification system to deal with the class imbalance problem in a big data scenario adding a cost-sensitive model to the MapReduce adaptation of the algorithm. In [17], a preliminary approach to make EUS work in a big data setting was developed following a twolevel parallelization model. As we have already mentioned, the greatest problem of this model was the small-sample size of the minority class, whose management with Hadoop framework was not fully automatic.

\section{Evolutionary Undersampling}

EUS [16] was developed as an extension of evolutionary prototype selection algorithms with special attention at the class imbalance problem [26]. In the case of EUS, the original objective of reducing the training set for the $k$ Nearest Neighbors $(k \mathrm{NN})$ slightly changes, giving more focus to the balancing of the dataset and to the correct identification of both classes of the problem in the subsequently used classifier. In order to obtain this new data subset, the instances of original dataset are encoded in a chromosome, which is evolved from randomly undersampled datasets until the best solution found cannot be further improved. The improvement is measured in terms of the fitness function, which in described afterwards.

In EUS, a binary chromosome is used to encode each possible solution. In the chromosome each bit represents the presence (1) or absence (0) of an instance in the training set. The search space is reduced by only considering the majority class instances for removal, including always all the minority class instances in the final dataset.

In order to rank the quality of the chromosomes a fitness function taking into account the balancing of the dataset and the expected performance of the selected instances is used. The performance is estimated by the leave-one-out technique using the $1 \mathrm{NN}$ classifier and is measured by the g-mean (defined in Eq. (2)). The complete fitness function is as follows:

$$
\text { fitness }_{\text {EUS }}= \begin{cases}\text { g-mean }-\left|1-\frac{n^{+}}{N^{-}} \cdot P\right| & \text { if } N^{-}>0 \\ \text { g-mean }-P & \text { if } N^{-}=0\end{cases}
$$

where $n^{+}$is the number of positive instances, $N^{-}$is the number of selected negative instances and $P$ is a penalization factor that focuses on the balance between both classes. $P$ is set to 0.2 as recommended by the authors, since it provides a good trade-off between both objectives.

As a search algorithm, the CHC evolutionary algorithm [27] is chosen due to its excellent balance between exploration and exploitation. $\mathrm{CHC}$ is an elitist genetic algorithm making use of the heterogeneous uniform cross-over (HUX) for the combination of two chromosomes. It also considers an incest prevention mechanism and instead of applying mutation, it carries out a reinitialization of the population when the evolution does not progress.

\section{EUS-EXTIMBBD: EVOLUTIONARY UNDERSAMPLING FOR EXTREMELY IMBALANCED BIG DATA}

In this section we describe the proposed scheme for EUS of extremely imbalanced big data. First, we motivate our proposal in Section III-A, stating the main drawbacks of our previously proposed scheme. Then, Section III give the details of the proposed model.

\section{A. Motivation}

The use of EUS in big data problems is interesting because it reduces the data size, in contradistinction to oversampling methods that generate even more data [20]. As a result, building classification models becomes a faster process. This data size reduction can also be achieved by random undersampling (RUS). However, RUS may discard important data of the majority class due to its random nature, while EUS guides the undersampling process to balance the dataset and preserve (or even improve) the accuracy in both classes.

In [17], we proposed a two-level parallelization scheme based on MapReduce and a windowing scheme for EUS. In the first level, a MapReduce process allowed us to divide the computational effort over different machines, creating different chunks of data. These subsets approximately contain the same number of instances and maintain the original imbalance ratio of the problem (if the original training set is properly shuffled). Thus, we run EUS for each subset, obtaining a balanced set that was later used to build a decision tree. The windowing scheme was applied on top of the MapReduce parallelization to reduce the computational time required by the fitness function of EUS. In order to do so, in each iteration of the evolutionary process, only one stratum of data was used to evaluate the population, which was changed in each iteration following a round-robin policy. Finally, after the building phase, a new MapReduce 
process was applied to classify the test set with the previously learned models. Figure 2 presents a flowchart of this model. In what follows, we denote this scheme as Evolutionary Undersampling for Imbalanced Big Data (EUS-ImbBD).

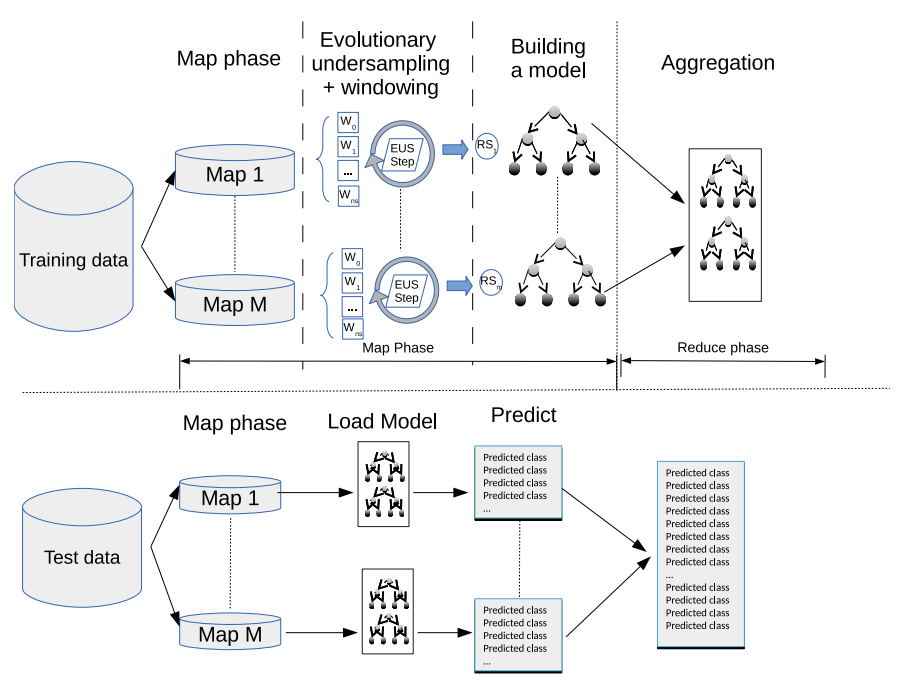

Fig. 2: Data flow of EUS-ImbBD.

This model suffers of two main drawbacks that motivates this work:

- Although EUS-ImbBD can handle very large datasets with the appropriate number of nodes, it may experience troubles to tackle extremely imbalanced problems in which the imbalance ratio is very high. In these cases, the amount of positive examples in the different chunks of the data created by the MapReduce process may not be sufficient (or even null) to guide an EUS process. This problem is known as the small sample size or lack of density problem. This is the main point motivating our works, since EUS-ImbBD becomes not totally scalable in this scenario.

- EUS-ImbBD requires to concatenate two MapReduce phases, to build a model and to classify a test set, respectively. Hadoop is inefficient with these types of models, whereas Spark allows us to avoid the startup costs. In Section IV the differences between Hadoop and Spark implementations of this scheme can be observed.

The aim of this paper is tackle both issues by designing an imbalance big data model, which relies on the flexibility an in-memory operations of Apache Spark.

\section{B. EUS-S-ExtImbBD: A Spark-based Design of Evolutionary Undersampling for Extremely Imbalanced Big Data}

This section introduces the proposed scheme in terms of multiple Spark distributed operations. Algorithm 1 shows the pseudo-code of the whole method with precise details of the functions utilized from Spark and Figure 3 summarizes the data flow of the algorithm. In the following, we describe the most significant instructions, enumerated from 1 to 12 .

Let trainFile be the training set stored in the HDFS as a single file. This file is formed of $h$ HDFS blocks that

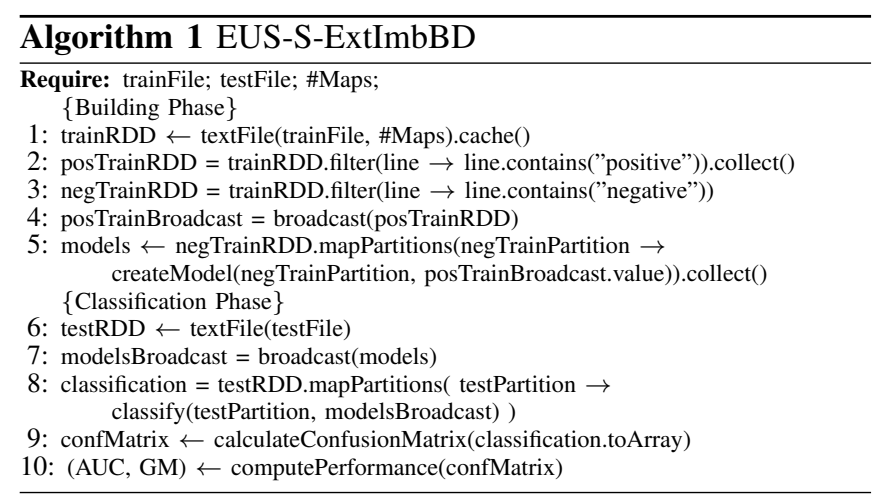

can be accessed from any computer. The building phase starts reading the whole trainFile set from HDFS as an RDD, splitting the dataset into an user-defined number of \#Map disjoint subsets (Instruction 1). This operation spreads the data across the computing nodes, caching the different subsets $\left(\mathrm{Map}_{1}, \mathrm{Map}_{2}, \ldots, \mathrm{Map}_{m}\right)$ into memory.

Next, we split this dataset into two subsets: positive set posTrainRDD and negative set negTrainRDD, which contain only positive and negative instances, respectively. The filter transformation provided by Spark is used for this purpose (Instructions 2 and 3).

Assuming that the number of positive instances fit inmemory, the whole posTrainRDD is collected and broadcast to all the computing nodes (Instruction 4). The broadcast function of Spark allows us to keep a read-only variable cached on the main memory of each machine rather than copying it with each tasks. Note that this can be a limitation of the current model, since if the number of positive instances is too high it may not fit in memory. However, this set of instances is stored only once in each node independently of the number of tasks executed in it. In the current real-world problems we are facing, with an extremely high imbalance ratios, this situation is difficult to be found. Anyway, in the future our aim is to further investigate problems with this scenario even though they may not suffer from the small-sample size problem due to the fact that more positive instances are available.

After that, the main map phase starts over the \#Map partitions (negTrainPartition) of negative set negTrainRDD (Instruction 5). The mapPartitions(func) transformation runs the function defined in Algorithm 2 on each block of the RDD concurrently. This function builds a model from the available data, i.e., a subset of negTrainPartition, and the whole posTrainBroadcast set. Depending on the dataset at hand, we may encounter two different situations:

- The number of positive examples is smaller or equal than the number of negative ones in the negTrainPartition. In this case, all the positive instances are added to the current subset trainingSet, obtaining a dataset with a lower imbalanced ratio than the original one (or almost balanced). In our experiments, when the resulting imbalanced ratio is greater or equal than 1.5, EUS is exclusively applied over the majority class. However, 

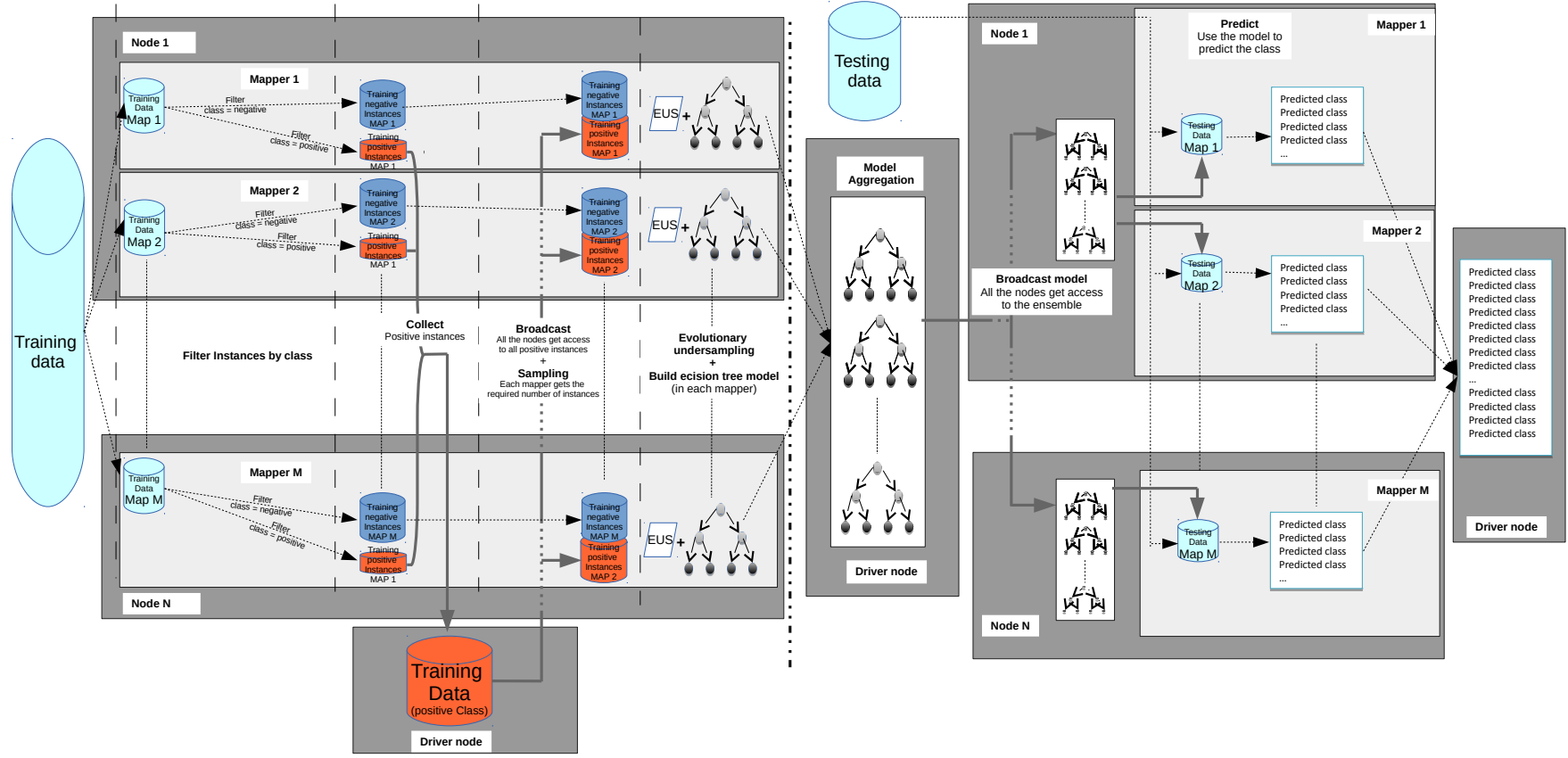

Fig. 3: Data flow of EUS-ExtImbBD.

if the problem is almost balanced $(I R<=1.5$ as suggested in the literature), instances from both classes are considered in the selection procedure, since the balancing looses importance in favor of an appropriate interaction between the instances of both classes (always maintaining a balance between their presence).

- When we the size of the positive class is larger than that of the negative one, we make use of a random subset of positive instances posTrainSubset from the whole positive set posTrain. Our aim is to balance the class distribution before applying EUS to reduce the size of both classes while focusing on maintaining a balance and obtaining the best performance as possible.

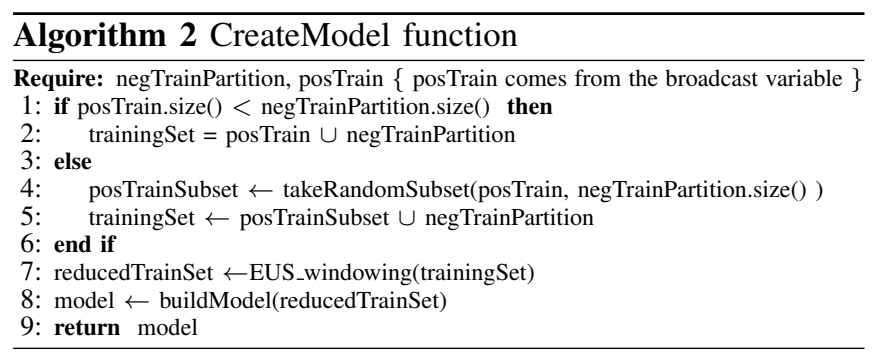

In both cases, EUS is applied with the windowing scheme. We refer the reader to [17] for more details on this respect. At the end of the EUS stage, a reduced and balanced set of instances (reducedTrainSet) is obtained. Then, the learning phase is carried out, which consists of building a decision tree. More specifically, we consider the well-known C4.5 algorithm [29] for its great behavior in classifier ensembles.

As a result of the map phase, we obtain \#Map decision trees that are returned to the driver and collected in Instruction 5 of Algorithm 1.
Then, the classification step estimates the class associated to each test example. Given that in big data problems the test set can also be very large, we assume this set is also stored in the HDFS as a single file (testFile), and read it as an RDD (Instruction 6). In order to classify the instances in each block, the models are broadcast to the main memory of all the computing nodes of the cluster (Instruction 7). A new map operation will tackle each subset of the test set. Algorithm 3 summarizes the operations that are carried out. Basically, the predictions in each block are estimated by the majority vote of the all the decision trees built in the previous phase (Instruction 8). Finally, the classification performed is collected in the driver and the performance measures are computed (Instructions 9 and 10).

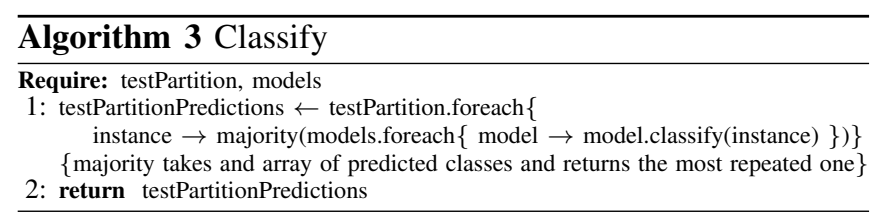

As a final remark, note that the EUS algorithm could be easily replaced by other data sampling approaches, without changing the general framework we propose in here.

\section{EXPERIMENTAL STUDY}

This section establishes the experimental setup (Section IV-A) and discusses the results obtained (Section IV-B.2).

\section{A. Experimental Framework}

In order to assess the proposed method for imbalanced big data, we consider two big data datasets with very high IR. The first dataset comes from the Evolutionary Big Data 
Competition ECBDL'14 [30], [6]. For this study, we consider two subsets of $25 \%$ and $50 \%$ of the instances, respectively. The second dataset corresponds to the KDD Cup 1999 data set, available in the UCI machine learning repository [31]. Since it contains multiple classes, we have formed several case studies from them, obtaining as a result two-class imbalanced problems. Specifically, we have taken the majority class (i.e., DOS) in comparison with the rest of the minority classes (i.e., PRB, R2L and $\mathrm{U} 2 \mathrm{R}$ ) in order to investigate the influence of different IRs. The data characteristics are summarized in Table I.

TABLE I: Data sets considered for the experimental study.

\begin{tabular}{crrrr}
\hline Data set & \#features & \#negative & \#positive & IR \\
\hline ECBDL'14 (50\%) & 631 & 17101086 & 344333 & 49 \\
ECBDL'14 (25\%) & 631 & 8550324 & 172386 & 49 \\
Kddcup DOS vs. PRB & 41 & 3883370 & 41102 & 94.48 \\
Kddcup DOS vs. R2L & 41 & 3883370 & 1126 & 3448.82 \\
Kddcup DOS vs. U2R & 41 & 3883370 & 52 & 74680.25 \\
\hline
\end{tabular}

In this study, independently of the technology (Hadoop or Spark) used, we distinguish between two different approaches to tackle imbalanced big data classification:

- EUS-ImbBD: EUS for Imbalanced Big Data, i.e., the model presented in [17], which consists of dividing the training set in a single MapReduce operation.

- EUS-ExtImbBD: EUS of Extremely Imbalanced Big Data problems, i.e., the scheme presented in this work, which considers the positive and negative training examples separately.

Originally, EUS-ImbBD was implemented under Hadoop (EUS-H-ImbBD), whereas EUS-ExtImbBD has been designed for Spark (EUS-S-ExtImbBD). However, in order to be able to perform a comparison between Hadoop and Spark technologies, we have implemented these models in both technologies, naming them as EUS-H-ExtImbBD and EUS$\mathrm{S}$-ImbBD, respectively. The comparison between Hadoop and Spark implementations will be done using KDD Cup dataset, as this was the one considered in the original work. Note that the application of EUS-ExtImbBD within Hadoop, one must manually pre-partition the training dataset into positive and negative sets, and the MapReduce stage is applied simply over the negative set, as it was done in [17], whereas the positive set has to be read in all the tasks (even if they are in the same node).

In addition to these models, we have implemented RUS in Spark under the original ImbBD scheme as a comparison algorithm, calling it RUS-S-ImbBD. Since RUS objective is to randomly balance the dataset eliminating negative class examples, this can be done in each mapper regardless of the number of instances from both classes in it, and hence it is not necessary to apply it under the scheme proposed for extremely imbalance data.

It is important to note that for the last two data sets presented in Table I, we have so few positive examples that the EUS-ImbBD approach cannot be applied, and EUSExtImbBD is required.

TABLE II: Parameter settings for the used methods.

\begin{tabular}{|c|c|}
\hline Method & Parameter values \\
\hline \multirow[t]{3}{*}{ EUS-ImbBD[17] } & MapReduce Building: Number Of Maps $=128 / 256 / 512 ;$ Number Of Reducers: 1 \\
\hline & Windowing in Majority Class $=$ Imbalanced Ratio \\
\hline & MapReduce Classification: Number Of Maps $=$ Same as Building phase; Number Of Reducers: 0 \\
\hline \multirow[t]{3}{*}{ EUS-ExtImbBD } & Building phase: Number Of Maps = 128/256/512/1024/2048; \\
\hline & Windowing in majority class $=\mathrm{IR}$; Windowing in both classes $=5$ \\
\hline & Classification phase: Number Of Maps = Same as Building phase; \\
\hline \multirow[t]{2}{*}{ RUS-S-ImbBD } & Building phase: Number Of Maps = 1024/2048; \\
\hline & Classification phase: Number Of Maps = Same as Building phase; \\
\hline
\end{tabular}

In our experiments we consider a 5-fold stratified crossvalidation model, meaning that we construct 5 random partitions of each dataset maintaining the prior probabilities of each class. Each fold, corresponding to $20 \%$ of the data is used once as test set, evaluated on a model trained on the combination of the 4 remaining folds. The reported results are taken as averages of the five partitions. To evaluate our model, we consider the AUC and g-mean measures recalled in Section II-B. Moreover, we evaluate the time requirements of the methods in two ways:

- Building time: we will quantify the total time in seconds spent by our method to generate the resulting learned model.

- Classification time: this refers to the time needed in seconds to classify all the instances of the test set with the given learned model.

We will also investigate how these measures are affected by modifying the number of maps. The experiments have been carried out on twelve nodes in a cluster: a master node and eleven computing nodes. Each one of these computing nodes has 2 Intel Xeon CPU E5-2620 processors, 6 cores per processor (12 threads), $2.0 \mathrm{GHz}$ and 64GB of RAM. The network is Gigabit ethernet (1Gbps). In terms of software, we have used the Cloudera's open-source Apache Hadoop distribution (Hadoop 2.6.0-cdh5.4.2) and Spark 1.5.1. A maximum of 216 concurrent tasks are available.

\section{B. Results and discussion}

This section presents and analyzes the results obtained in the experimental study. We divide this section into two parts: Subsection IV-B.1 briefly compares Hadoop and Spark implementations, and Subsection IV-B.2 deeply analyzes the performance of the proposed approach in a larger problem.

1) Comparing Hadoop vs. Spark: The goal of this subsection is to compare the Hadoop and Spark technologies in terms of efficiency, when they implement the same model. To do this, we focus on the three different variants of the Kddcup dataset. The EUS-ImbBD approach is used for DOS vs. PRB dataset, whereas EUS-ExtImbBD is applied in the other two Kddcup versions as explained before. Table III shows the runtime required in both building and classification phases, depending on the technology used, as well as the percentage of improvement obtained with Spark over Hadoop.

According to this table, we can state that: 
TABLE III: Running times obtained by the Hadoop and Spark implementations of EUS-ImbBD and EUS-ExtImbBD.

\begin{tabular}{c|r|rr|rrr|r}
\hline & & \multicolumn{2}{|c|}{ Hadoop-based } & \multicolumn{2}{c|}{ Spark-based } & \multicolumn{2}{c}{ Spark improvement } \\
Dataset & No. of maps & Build time (s) & Classif. time (s) & Build time(s) & Classif. time (s) & Build time & Classif. time \\
\hline Kddcup DOS vs. PRB & 128 & 422.4786 & 34.264 & 297.5048 & 0.2942 & $29.58 \%$ & $99.14 \%$ \\
& 256 & 240.4662 & 36.7934 & 143.3428 & 0.3566 & $40.39 \%$ & $99.03 \%$ \\
& 512 & 156.4354 & 48.424 & 87.0195 & 0.2739 & $44.37 \%$ & $99.43 \%$ \\
\hline Kddcup DOS vs. R2L & 128 & 444.7252 & 31.7255 & 320.8192 & 0.0876 & $27.86 \%$ & $99.72 \%$ \\
& 256 & 266.2424 & 36.1147 & 187.4562 & 0.1024 & $29.59 \%$ & $99.72 \%$ \\
& 512 & 178.8536 & 42.0057 & 148.319 & 0.1371 & $17.07 \%$ & $99.67 \%$ \\
\hline Kddcup DOS vs. U2R & 128 & 459.6002 & 31.8436 & 340.2297 & 0.0986 & $25.97 \%$ & $99.69 \%$ \\
& 256 & 248.1038 & 35.5862 & 193.0784 & 0.1081 & $22.18 \%$ \\
& 512 & 152.3752 & 46.6194 & 101.683 & 0.1275 & $33.27 \%$ & $99.70 \%$ \\
& & & &
\end{tabular}

- Even though the same EUS process has been applied in both technologies, the Spark-based implementation has always reported a faster runtime in both building and classification phases. It is remarkable that applying EUS-ExtImbBD, Spark is still much faster, although the Hadoop version already started from two manually partitioned datasets (whose added cost is not included in the runtime). Moreover, it is specially significant the reduction in terms of classification, due to the startup costs of Hadoop MapReduce.

- From the flexibility point of view, Spark has allowed us to apply the whole procedure in a single program, while in Hadoop, multiple MapReduce programs needs to be chained. Hence, Spark is more versatile.

In conclusion the use of Spark has provided us a greater flexibility and efficiency. In the next experiments, we will only consider Spark to tackle larger datasets.

2) Analysis of the performance: To analyze the performance of the proposed EUS-S-ExtImbBD scheme, we focus on the ECBLD'14 dataset. Tables IV and V show the results obtained by our proposal in comparison to the previous alternative (EUS-S-ImbBD) and random undersampling (RUSS-ImbBD) in ECBLD'14 (25\%) and ECBLD'14 (50\%), respectively. All of these techniques are implemented under Apache Spark for a faster computation. The averaged AUC, g-mean, building and classification runtime are presented, depending on the number of mappers used (\#Maps).

TABLE IV: Results obtained in ECBLD'14 (25\%)

\begin{tabular}{l|c|rr|cc}
\hline Method & \#Maps & Build time(s) & Classif. time(s) & AUC & GM \\
\hline EUS-S-ImbBD & 4096 & 243.8191 & 0.3376 & 0.5600 & 0.3714 \\
& 2048 & 420.2240 & 0.6048 & 0.4885 & 0.3886 \\
& 1024 & 790.6699 & 0.5408 & 0.6648 & 0.6582 \\
\hline EUS-S-ExtImbBD & 4096 & 1156.8111 & 2.0065 & 0.6644 & 0.6644 \\
& 2048 & 1775.9417 & 3.0516 & 0.6655 & 0.6650 \\
& 1024 & 3268.2816 & 4.9365 & 0.6733 & 0.6717 \\
\hline RUS-S-ImbBD & 4096 & 276.0484 & 0.3616 & 0.6319 & 0.6027 \\
& 2048 & 233.7217 & 0.4657 & 0.4595 & 0.4426 \\
& 1024 & 224.8443 & 0.5298 & 0.6564 & 0.6502 \\
\hline
\end{tabular}

From these tables we can highlight several factors:

- When the number of maps is increased, we can observe
TABLE V: Results obtained in ECBLD'14 (50\%)

\begin{tabular}{l|c|rr|cc}
\hline Method & \#Maps & Build time(s) & Classif. time(s) & AUC & GM \\
\hline EUS-S-ImbBD & 8192 & 497.0958 & 0.4999 & 0.5556 & 0.3572 \\
& 4096 & 775.2016 & 0.5531 & 0.4821 & 0.4276 \\
& 2048 & 1476.3512 & 0.7878 & 0.6674 & 0.6645 \\
\hline EUS-S-ExtImbBD & 8192 & 2181.5089 & 3.5404 & 0.6641 & 0.6640 \\
& 4096 & 3456.5938 & 6.0428 & 0.6662 & 0.6657 \\
& 2048 & 6433.8072 & 9.4064 & 0.6731 & 0.6704 \\
\hline RUS-S-ImbBD & 8192 & 557.4869 & 0.5540 & 0.6319 & 0.6066 \\
& 4096 & 518.1471 & 1.0960 & 0.4659 & 0.4339 \\
& 2048 & 483.7361 & 1.0960 & 0.6651 & 0.6622 \\
\hline
\end{tabular}

how the EUS-S-ImbBD scheme falls into the small sample size problem. The reduction of the number of positive examples in the different maps makes AUC and GM measures to drastically drop when more maps are required. AUC values are sometimes even lower than 0.5 meaning that an inappropriate model has been learned (worse than random guessing).

- On the other side, the proposed EUS-S-ExtImbBD has been able to avoid this issue by using a higher number positive examples in each map. We can observe how the decrease in precision when the number of maps is increased is much smoother in this case. Nevertheless, since EUS is dealing with a larger number of examples in each map in comparison with EUS-S-ImbBD, the total runtime required becomes higher for this proposal.

- Comparing with the application of a simple random undersampling (RUS-S-ImbBD), we can observe that the capabilities of EUS are maintained and that it is able to outperform RUS in terms of precision. Of course, the time required by RUS is much lower, since it does not involve any heuristic mechanism to select elements from the negative class.

- Note that the number of concurrent tasks in the used cluster is 216. Thus, we cannot expect a linear speedup in the runtime required by RUS. We do appreciate a linear complexity reduction for EUS due to the quadratic complexity of this problem.

- Finally, the small sample size problem in EUS-SImbBD and RUS-S-ImbBD can be clearly observed 
attending at their instability when addressing the same problem with different number of mappers. Notice that after the sampling process a $\mathrm{C} 4.5$ decision tree is built, and not only its building process is affected by the number and class of the examples selected but also its pruning may vary (which can explain the instability of these models).

Attending at these results, one can conclude that the new EUS model for addressing extremely imbalanced problems with Spark overcome the problems of our previous alternatives. Moreover, the flexibility of Spark has allowed us to make a simple and fully automatic implementation of the proposed model.

\section{CONCLUDING REMARKS}

In this contribution we have devised a big data scheme to deal with the rare situation where data scarcity (of a particular class) remains to be a problem despite the vast quantity of available data. The application of the proposed strategy enables EUS to be applied on big datasets with extremely skewed class distributions by an effective use of the available data. Our implementation is based on multiple parallel operations and takes advantage of the in-memory operations provided by Apache Spark. Our experimental study shows the benefits of using Spark as parallelization technology and the advantages of our new framework to soften the lack of density issue presented in these extremely imbalanced problems. As future work, we consider the development of efficient big data strategies that can deploy preprocessing mechanisms such as hybrid oversampling/undersampling approaches in the big data context. Regarding the proposed method, techniques to deal with extremely imbalanced problems where the positive class does not fit in the main memory of the computing nodes will be studied.

\section{REFERENCES}

[1] C. Lynch, "Big data: How do your data grow?" Nature, vol. 455, no. 7209 , pp. 28-29, 2008.

[2] M. Minelli, M. Chambers, and A. Dhiraj, Big Data, Big Analytics: Emerging Business Intelligence and Analytic Trends for Today's Businesses (Wiley CIO), 1st ed. Wiley Publishing, 2013.

[3] J. Dean and S. Ghemawat, "Mapreduce: simplified data processing on large clusters," Communications of the ACM, vol. 51, no. 1, pp. 107-113, Jan. 2008.

[4] S. Ghemawat, H. Gobioff, and S.-T. Leung, "The google file system," in Proceedings of the nineteenth ACM symposium on Operating systems principles, ser. SOSP '03, 2003, pp. 29-43.

[5] A. Srinivasan, T. Faruquie, and S. Joshi, "Data and task parallelism in ILP using mapreduce," Machine Learning, vol. 86, no. 1, pp. 141-168, 2012.

[6] I. Triguero, S. del Río, V. López, J. Bacardit, J. M. Benítez, and F. Herrera, "ROSEFW-RF: The winner algorithm for the ecbdl'14 big data competition: An extremely imbalanced big data bioinformatics problem," Know.-Based Syst., vol. 87, no. C, pp. 69-79, Oct. 2015.

[7] A. Fernández, S. Río, V. López, A. Bawakid, M. del Jesus, J. Benítez, and F. Herrera, "Big data with cloud computing: An insight on the computing environment, mapreduce and programming frameworks," WIREs Data Mining and Knowledge Discovery, vol. 4, no. 5, pp. 380409, 2014.

[8] K. Grolinger, M. Hayes, W. Higashino, A. L'Heureux, D. Allison, and M. Capretz, "Challenges for mapreduce in big data," in Services (SERVICES), 2014 IEEE World Congress on, June 2014, pp. 182-189.
[9] Y. Low, J. Gonzalez, A. Kyrola, D. Bickson, C. Guestrin, and J. M. Hellerstein, "Graphlab: A new parallel framework for machine learning," in Conference on Uncertainty in Artificial Intelligence (UAI), Catalina Island, California, July 2010.

[10] Y. Bu, B. Howe, M. Balazinska, and M. D. Ernst, "Haloop: Efficient iterative data processing on large clusters," Proc. VLDB Endow., vol. 3 , no. 1-2, pp. 285-296, Sep. 2010.

[11] M. Zaharia, M. Chowdhury, T. Das, A. Dave, J. Ma, M. McCauley, M. J. Franklin, S. Shenker, and I. Stoica, "Resilient distributed datasets: A fault-tolerant abstraction for in-memory cluster computing," in Proceedings of the 9th USENIX conference on Networked Systems Design and Implementation. USENIX Association, 2012, pp. 1-14.

[12] V. López, A. Fernández, S. García, V. Palade, and F. Herrera, “An insight into classification with imbalanced data: Empirical results and current trends on using data intrinsic characteristics," Information Sciences, vol. 250, no. 0, pp. 113 - 141, 2013.

[13] G. Weiss, "Mining with rare cases," in Data Mining and Knowledge Discovery Handbook. Springer, 2005, pp. 765-776.

[14] M. Galar, A. Fernández, E. Barrenechea, H. Bustince, and F. Herrera, "A review on ensembles for the class imbalance problem: Bagging, boosting-, and hybrid-based approaches," IEEE Transactions on Systems, Man, and Cybernetics, Part C: Applications and Reviews, vol. 42, no. 4, pp. 463-484, 2012.

[15] M. Galar, A. Fernández, E. Barrenechea, and F. Herrera, "Eusboost: Enhancing ensembles for highly imbalanced data-sets by evolutionary undersampling," Pattern Recognition, vol. 46, no. 12, pp. 3460-3471, 2013.

[16] S. García and F. Herrera, "Evolutionary under-sampling for classification with imbalanced data sets: Proposals and taxonomy," Evolutionary Computation, vol. 17, no. 3, pp. 275-306, 2009.

[17] I. Triguero, M. Galar, S. Vluymans, C. Cornelis, H. Bustince, F. Herrera, and Y. Saeys, "Evolutionary undersampling for imbalanced big data classification," in Evolutionary Computation (CEC), 2015 IEEE Congress on, May 2015, pp. 715-722.

[18] A. H. Project, "Apache hadoop," 2013. [Online]. Available: http://hadoop.apache.org/

[19] T. Fawcett, "An introduction to roc analysis," Pattern recognition letters, vol. 27, no. 8, pp. 861-874, 2006.

[20] S. del Río, V. López, J. Benítez, and F. Herrera, "On the use of mapreduce for imbalanced big data using random forest," Information Sciences, vol. 285, pp. 112-137, 2014

[21] G. Batista, R. Prati, and M. Monard, "A study of the behavior of several methods for balancing machine learning training data," $A C M$ Sigkdd Explorations Newsletter, vol. 6, no. 1, pp. 20-29, 2004.

[22] N. Chawla, K. Bowyer, L. Hall, and W. Kegelmeyer, "SMOTE: synthetic minority over-sampling technique," arXiv preprint arXiv:1106.1813, 2011.

[23] L. Breiman, "Random forests," Machine learning, vol. 45, no. 1, pp. 5-32, 2001.

[24] V. López, S. del Río, J. Benítez, and F. Herrera, "Cost-sensitive linguistic fuzzy rule based classification systems under the mapreduce framework for imbalanced big data," Fuzzy Sets and Systems, vol. 258, pp. 5-38, 2014

[25] H. Ishibuchi, T. Nakashima, and M. Nii, Classification and modeling with linguistic information granules. Springer, 2005.

[26] S. García, J. Derrac, J. Cano, and F. Herrera, "Prototype selection for nearest neighbor classification: Taxonomy and empirical study," IEEE Transactions on Pattern Analysis and Machine Intelligence, vol. 34, no. 3, pp. 417-435, 2012.

[27] L. J. Eshelman, "The CHC adaptive search algorithm: How to have safe search when engaging in nontraditional genetic recombination," in Foundations of Genetic Algorithms, G. J. E. Rawlins, Ed. San Francisco, CA: Morgan Kaufmann, 1991, pp. 265-283.

[28] I. Triguero, D. Peralta, J. Bacardit, S. Garcia, and F. Herrera, "A combined mapreduce-windowing two-level parallel scheme for evolutionary prototype generation," in Evolutionary Computation (CEC), 2014 IEEE Congress on, July 2014, pp. 3036-3043.

[29] J. R. Quinlan, C4.5: programs for machine learning. San Francisco, CA, USA: Morgan Kaufmann Publishers, 1993.

[30] "ECBDL14 dataset: Protein structure prediction and contact map for the ECBDL2014 big data competition," 2014. [Online]. Available: http://cruncher.ncl.ac.uk/bdcomp/

[31] A. Frank and A. Asuncion, "UCI machine learning repository," 2010. [Online]. Available: http://archive.ics.uci.edu/ml 